\title{
Hemorrhagic fever in Saudi Arabia: challenge to public health, effective management and future considerations
}

\author{
Taha A Kumosani ${ }^{1,2,3}$, Abdulrahman L Al-Malki ${ }^{1,2,4}$, Syed S Razvi ${ }^{1,5}$, Maha J Balgoon ${ }^{1}$, \\ Mohammed Kaleem ${ }^{1,4}$, Etimad A Huwait ${ }^{1,3}$, Maryam A Alghamdi ${ }^{1}$, Soonham S Yaghmoor ${ }^{2,3}$, \\ Khalid O Abualnaja ${ }^{1,4}$, Elie K Barbour ${ }^{1,2,6}$, Khalid A AL-Madani ${ }^{7}$, Majdi H AlToukhi ${ }^{8}$, \\ Afnan T Kumosani ${ }^{9}$, Said S Moselhy ${ }^{10}$
}

1. Department of Biochemistry, Faculty of Science, King Abdulaziz University, Jeddah, Saudi Arabia.

2. Experimental Biochemistry Unit, King Fahd Medical Research Center, King Abdulaziz University, Jeddah, Saudi Arabia.

3. Production of Bioproducts for Industrial Applications Research Group, King Abdulaziz University, Jeddah, Saudi Arabia.

4. Bioactive Natural Products Research Group, King Abdulaziz University, Jeddah, Saudi Arabia.

5. Math and Science Department, Community College of Qatar.

6. Director of R and D Departement, Opticon Hygiene Consulting, Oechsli7,8807 Freienbach, Switzerland.

7. Consultant in Clinical Nutrition,Member of the Boardof ILSI Middel East, Member of the Board of Saudi Society for Food and Nutrition. Advance Clinics, Jeddah, Saudi Arabia.

8. General Supervisor Health and Environmental Department and Consultant, Public Health and Infectious Diseases.

9. Department of pathology and Laboratory Medicine, Blood Bank Transfusion medicine, King Abdulaziz Medical City, Jeddah, National Guards, Jeddah, 21423.

10. Biochemistry Department, Faculty of Science, Ain shams University, Cairo, Egypt.

\begin{abstract}
Background: Viral hemorrhagic fevers (VHF) refers to a group of febrile illnesses caused by different viruses that result in high mortality in animals and humans. Many risk factors like increased human-animal interactions, climate change, increased mobility of people and limited diagnostic facility have contributed to the rapid spread of VHF.

Materials: The history of VHFs in the Saudi Arabian Peninsula has been documented since the $19^{\text {th }}$ century, in which many outbreaks have been reported from the southwestern region of Saudi Arabia. Despite presence of regional network of experts and technical organizations, which expedite support and respond during outbreaks, there are some more challenges that need to be addressed immediately. Gaps in funding, exhaustive and inclusive response plans and improved surveillance systems are some areas of concern in the region which can be dealt productively. This review primarily focusses on the hemorrhagic fevers that are caused by three most common viruses namely, the Alkhurma hemorrhagic fever virus, Rift valley fever virus, and Dengue fever virus.

Conclusion: In summary, effective vector control, health education, possible use of vaccine and concerted synchronized efforts between different government organizations and private research institutions will help in planning effective outbreak-prevention and response strategies in future.

Keywords: Viral fever; hemorrhagic fever (VHF); Saudi Arabia; challenges; management; future considerations.

DOI: https://dx.doi.org/10.4314/ahs.v20i3.17

Cite as: Kumosani TA, Al-Malki AL, Razvi SS, Balgoon MJ, Kaleem M, Huwait EA, et al. Hemorrbagic fever in Saudi Arabia: challenge to public health, effective management and future considerations. Afri Health Sci. 2020;20(3): 1153-1163. bttps:// dx.doi.org/10.4314/ahs. v20i3.17
\end{abstract}

\section{Corresponding author:}

Said S Moselhy,

Biochemistry Department, Faculty of Science,

Ain shams University, Cairo, Egypt.

Email: moselhy6@hotmail.com

\section{Background}

Viral hemorrhagic fever (VHF) refers to a complex etiology of pathological manifestations and symptoms characterised by hemorrhagic indications with febrile illness and vascular damage leading to high mortalities ${ }^{1,2}$. The nature of the symptoms depends upon the caus- 
ative agent of VHF; generally, the symptoms include high fever, vomiting, diarrhoea, dizziness, nausea, loss of strength and muscle aches. The incubation time of the disease varies according to the etiology of VHF, ensuing in a range of 2-21 days. In severe cases, the VHF may also induce blood coagulation, leading to petechial or ecchymotic bleeding beneath the skin, conjunctivitis, pulmonary haemorrhage, pleural effusion, pneumonia, and acute respiratory distress.

The most common VHF-viruses that have been epidemic belong to four families; namely, Flaviviridae (Alkhurma hemorrhagic fever-AHF and Dengue fever and yellow fever), Bunyaviridae (Rift Valley fever-RVF, Crimean-Congo hemorrhagic fever-CCHF, Arenaviridae (Lassa fever) and Filoviridae (Ebola fever). It is interesting to note that all these diseases are caused by RNA viruses possessing a lipid bilayered envelope recovered from the cell membrane of the host. The tenacity of these viruses in the environment depends solely on the natural reservoir hosts (insects and animals). These viruses are transmitted from an infected person to a healthy individual by direct contact or via the infected body fluids.

The history of the hemorrhagic fever in the region of Arabian Peninsula dates back to the $19^{\text {th }}$ century, documenting a significant outbreak of Dengue-like disease. Nevertheless, the widespread frequency of VHFs has been reported since the mid-1990s, witnessing a sharp rise in new cases during the last two decades, prompting different health agencies and organisations to im- plement strict measures and policies ensuring effective management of the VHFs.

This manuscript mainly focusses on the etiology, clinical challenge, modes of transmission, effective management, and future considerations in control of the three most common VHFs namely, Alkhurma hemorrhagic fever virus, Rift valley fever virus, and Dengue fever virus.

\section{Alkhurma hemorrhagic fever (AHF):}

Alkhurma hemorrhagic fever virus (AHFV) was initially isolated from a butcher in the year of 1995, who slaughtered a sheep in the Alkhurma region and was admitted at Soliman Fakeeh Hospital, with hysterical symptoms of fatal hemorrhagic fever ${ }^{3}$. Accordingly, the name 'Alkhurma' was later approved by the International Committee for Taxonomy of Viruses, relating the nomenclature of this virus to the village 'Alkhurma' in the Kingdom of Saudi Arabia ${ }^{4}$. Subsequently, many new cases followed in the KSA, with similar symptoms and clinical manifestation ${ }^{5}$. It is worth noting that the national epidemiology of the AHFV is yet to be uncovered in this country ${ }^{4}$. Fig. 1 showed the areas in KSA that were affected by AHFV; this Figure was constructed based on the limited targeted surveillance, in which the confirmed cases in regions affected by AHFV are highlighted in red colour, while the possible diagnosis, based only on positive serology of investigated cases, are highlighted in blue.

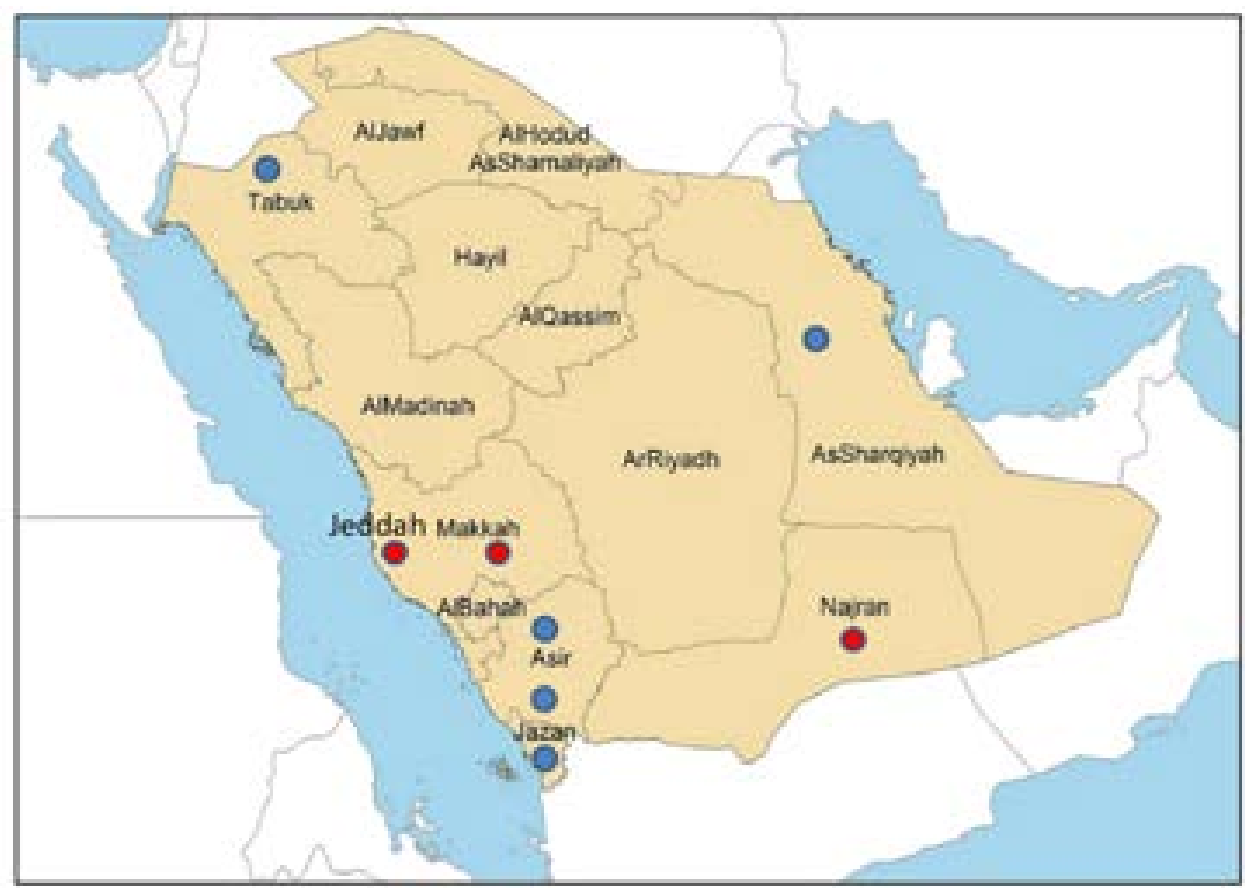

Fig. 1 Regions in the Kingdom of Saudi Arabia, where people

were infected with Alkhurma Hemorrhagic Fever Virus (AHFV). Red circles designate the areas with confirmed AHFV cases, while blue circles designate the areas of serologically positive individuals ${ }^{17}$. 


\section{The diversity of AHFV}

AHFV was the first discovered tick-borne virus associated with apparent clinical illness in the whole Arabian Peninsula ${ }^{6}$. AHFV belongs to the family Flaviviridae, which is comprised of more than 70 viruses that are transmitted by either ticks or mosquitoes. It is interesting to note that AHFV is a genotypic variant of Kyasanur Forest Disease Virus (KFDV) that caused fatal hemorrhagic fever in the state of Karnataka, In$\mathrm{dia}^{7}$. This relatedness was confirmed by analysing the data obtained from the whole genome sequencing of AHFV. In fact, both KFDV and AHFV showed an $89 \%$ sequence homology $y^{8,9}$.

\section{Modes of transmission}

There is a strong entomological, virological, epidemiological and phylogenetic evidence that AHFV is a tickborne flavivirus ${ }^{9-11}$ ). Based on such data, the reservoirs/ vectors of AHFV have been identified as a hard tick (Hyalomma dromedarii) and a soft tick (Ornithodoros savignyi $)^{10,12}$. AHFV was isolated from these ticks, that also exist in the neighbouring areas. These ectoparasite ticks were found on the livestock of Makkah and $\mathrm{Na}$ jran regions, incriminating it as a responsible vector of AHFV. Future investigation should involve the sheep and camels infested by these ectoparasites to study there if they have a role in amplification and transmission of the etiologic agent of this disease. The available epidemiological data in KSA suggest that the human consumption of raw milk, the tick bites, and contact with the blood of slaughtered animals could be the source of transmission of these viruses ${ }^{7}$. It is documented that $42 \%$ of the 233 confirmed human cases with AHFV infection were either butchers, abattoir workers, shepherds or just involved in livestock business, while only $33 \%$ of these patients had no contact with the livestock, suggesting an alternative route of transmission, other than those listed above 4 . It is worth noting that there is no data confirming a person-to-person transmission of $\mathrm{AHFV}^{13}$. Besides, there is limited data pointing at the role of mosquitoes in this transmission, that lead to an absence of its implication ${ }^{14}$. However, this virus had an ability to propagate in mosquitoes, leading to a hypothesis that it could be involved as a vector in this viral transmission ${ }^{14}$.

\section{Clinical Challenges}

All the initial incidents reported showed symptoms of hemorrhagic fevers, ranging from severe to fatal, increasing the liver enzymes, causing thrombocytopenia, leucopenia, headaches, generalised body aches, elevated blood urea and creatinine phosphokinase, manifested in an apparent challenge to the public health in Saudi Arabia ${ }^{2}$. Some presentations also included skin rash and encephalitis. A descriptive cohort study reported 148 suspected cases in the region of Najran province from first of August 2003 through $31^{\text {st }}$ of December 2009. Seventy-eight cases were confirmed by laboratory tests, in which $50 \%$ of these occurred during summer ${ }^{15}$. The clinical symptoms included fever $(100 \%)$, arthralgia $(83.3 \%)$, anorexia $(82.1 \%)$, nausea and vomiting (71.8\%), chills $(60.3 \%)$, retro-orbital pain $(55.1 \%)$ and diarrhoea $(51.3 \%)$, beside an increase in liver enzymes ${ }^{15}$. In a different study, twenty confirmed cases were reported in Makkah with febrile flu-like symptoms and hepatitis (100\%), hemorrhagic manifestations (55\%) and encephalitis $(20 \%)^{16}$. Moreover, the cases reported earlier showed a range of $25-30 \%$ fatalities ${ }^{16,17}$; Subsequently, the casualty was reduced to as low as $1.3 \%$ in another investigation of 281 cases that occurred between the years 2011 to $2014^{17}$. Nonetheless, the primary data could be biased, due to the fact that it included the most severe cases, while possibly excluding the mild and asymptomatic AHF. In the year 2010, only 81 cases were documented in the KSA, with only two fatalities ${ }^{6}$. The existence of such a low index of AHF incrimination, in some parts of the country, is most likely due to the inadequately implemented diagnostic procedures, resulting in unreliable differential diagnosis among the cross-reactive flaviviruses; for example, there is a difficulty to differentiate between the co-endemic AHFV and Dengue, in the absence of the technology needed in such a differential diagnosis ${ }^{6}$. The details of suspected and confirmed cases of AHFV that occurred in Saudi Arabia during the last two decades are presented in Table 1. 
Table 1: Number of suspected and confirmed cases of Alkhurma hemorrhagic fever virus in the Kingdom of Saudi Arabia in the last two decades.

\begin{tabular}{cccccc}
\hline $\begin{array}{c}\text { No of } \\
\text { suspected } \\
\text { cases }\end{array}$ & $\begin{array}{c}\text { No of } \\
\text { confirmed } \\
\text { cases }\end{array}$ & $\begin{array}{c}\text { No of } \\
\text { fatalities }\end{array}$ & Year & City & Reference \\
\hline 11 & 11 & 4 & $1994-99$ & Makkah or Jeddah & 7 \\
10 & 8 & 2 & 1997 & South Jeddah & 41 \\
37 & 20 & NA' & $2001-03$ & Mecca & 42 \\
148 & 78 & NA & $2003-09$ & Najran & 15 \\
197 & 13 & NA & 2009 & Jizan & 43 \\
59 & 59 & NA & 2009 & Jeddah and Najran & 4 \\
81 & 81 & 2 & 2010 & Jeddah, Najran, Jizan & 4 \\
& & & & and Mecca & \\
& 93 & NA & 2011 & Jeddah, Najran Jizan, & Makkah and Taif \\
\hline${ }^{1} \mathrm{NA}=$ Not Applicable & & & &
\end{tabular}

\section{Effective management and future considerations}

The AHF seems geographically confined. There should be a priority in the future perspective to develop an autogenous vaccine to protect against local AHFV strains. Educational public awareness, especially among the workers of livestock industry, could reduce significantly the incidence of AHF. Avoiding the consumption of AHFV-contaminated raw and unpasteurised milk will prevent the human consumer from contracting these viruses through this liquid food ${ }^{6}$. Obligatory use of coveralls and tick repellant by abattoir workers is a must to reduce their infection rate. The use of permethrin-impregnated clothing was found highly useful in preventing tick bites, thus intercepting against the tickmode of transmission ${ }^{18}$.

The future consideration by the KSA to adopt the One health strategy advocated by the World Animal Health Organisation and the World Health Organisation aimed at protecting the three arms of the triangle namely, humans, animals, and environment, which is expected to pave the way for better effective management of AHFV $^{19}$. Adopting this strategy will require an urgent collaborative effort among researchers, clinicians, veterinarians and specialists to approve a plan of action to control these zoonotic diseases. It is worth noting that three stages were suggested for development of vaccines to protect against AHFV namely, first, vaccines for humans and economically valuable animals, second, vaccines for domestic animals to reduce the animal to human transmission, and third, vaccines for wild animals ${ }^{19}$.

\section{Rift Valley fever (RVF)}

The data obtained from phylogenetic studies suggest that the RVF was introduced into Saudi Arabia from Kenya $^{20}$. In a separate study, Miller and colleagues compared the genomic sequence of Rift Valley Fever Virus (RVFV), isolated from the mosquitoes in Asir region, to different geographical isolates, concluding a high relatedness between the Saudi Arabian isolate and that of Madagascar and Kenyan strains ${ }^{20}$. This explains the hypothesis that the RVFV originated from the livestock imported from East Africa, and transmitted by mosquitoes drifted by strong wind gust over the Red Sea and the Gulf of Aden. The first outbreak of RVF was reported in southwestern Saudi Arabia, at Al-Humayrah village of Al-Ridah district near to lake Al-sad; this outbreak occurred in September 2000, involving around 883 confirmed cases and 123 deaths $^{21}$. Strict measures were taken by Saudi Ministry of Health $(\mathrm{MOH})$, which included mass immunisation of ruminant animals, 
eradication of infected animals, vector control, ban on importing livestock from East Africa, awareness campaign, and targeted surveillance. These efforts resulted in absence of animal clinical cases and mortalities by May 2001 and until the present time ${ }^{21}$.

\section{The diversity of the RVF Virus}

RVF is an acute infectious disease which is caused by an arbovirus, belonging to the family Bunyaviridae ${ }^{21}$. It is one of the significant zoonotic diseases which was initially confined to Africa, with later pieces of evidence of an expanded epizootiology to other geographical regions. The frequency of RVF affected animals were $65.6 \%$ in Jazan region, $27 \%$ in Tihamat Asir, and 7.5\% in Tihamah Makkah. A high seropositive prevalence was found in sheep $(10 \%)$, followed by $8 \%$ in goats, $1.2 \%$ in cattle and $1.3 \%$ in camels ${ }^{21}$.

\section{Modes of transmission}

A broad range of blood-sucking arthropods has been reported in Saudi Arabia, including many species of mosquitoes, classified under the genera Culex, Anopheles, Aedes, Culista, and Uranotaenia ${ }^{22}$. Numerous species of these are the vectors of RVFV. In a study performed by Jupp et al., female mosquitoes were collected in large numbers from the affected areas and examined for the presence of $\mathrm{RVFV}^{22}$. The RVFV was present in two species of mosquito, namely Aedes vexans arabiensis and Culex (culex) triteniorynchus, both of these were found in abundance in the epizootic region.

Moreover, both mosquito species were proven to feed on the blood of the sheep and humans in the epizootic area. Figure 2 shows the life cycle of RVFV. It is worth noting that the emergence and dissemination of vector-borne diseases depend considerably on the climate that is appropriate for vector survival in the area where the animals are reared ${ }^{21}$.

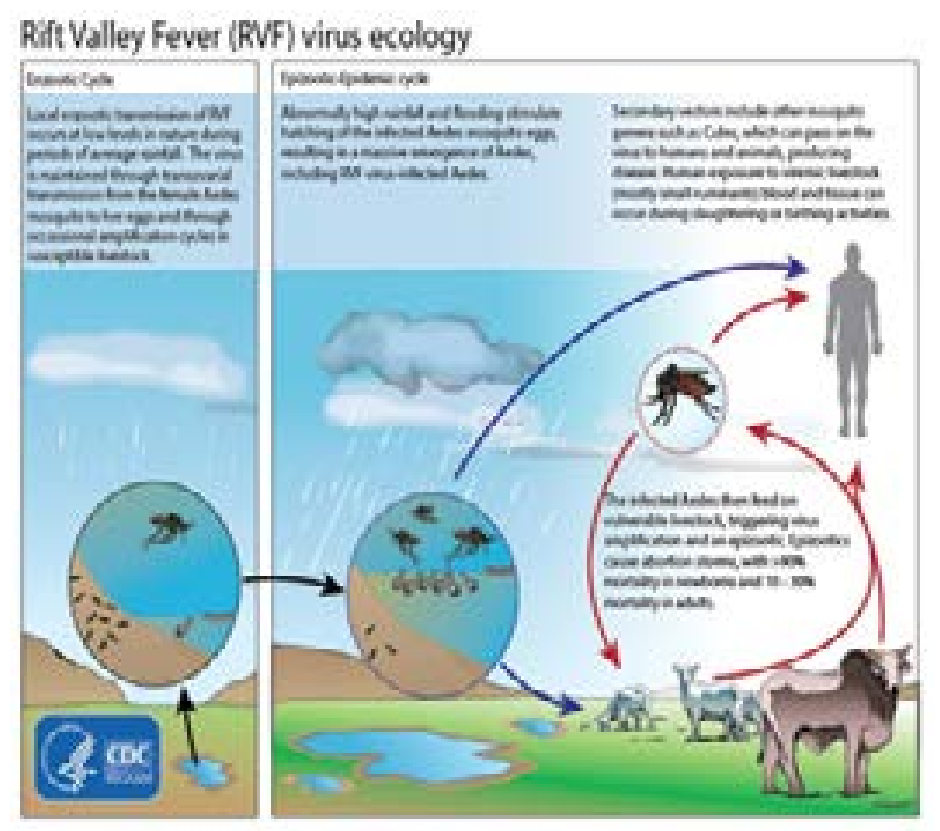

Fig. 2: Ecology of Rift Valley Fever (RVF). Courtesy of the Center for Disease Control, Atlanta, Georgia.

\section{Clinical challenges}

The data obtained from Jazan General Hospital showed the occurrence of severe and acute cases of RVF. The bracketed frequency of patients, manifesting different conditions, were: Liver failure $(75 \%)$, acute renal failure (41\%), hemorrhagic fever $(20 \%)$ and late manifestations of macular and paramacular retinitis $(10 \%)$ accompanied by meningoencephalitis $(4 \%)^{23}$. The primary causes of mortalities were severe anaemia, hepato-renal failure, shock due to lack of blood flow, and disseminated intravascular clotting. Another study reported the pres- ence of hemorrhagic fever, nausea, diarrhoea, vomiting and abdominal pain as primary manifestations in RVF affected patients of Saudi Arabia ${ }^{24}$. RVF also caused a high prevalence of ocular implications in affected patients, including retinal bleeding (40\%), alterations in vitreous viscosity $(26 \%)$, oedema in optic disc $(15 \%)$, and retinal vasculitis $(7 \%)^{25}$. Subsequently, these lesions affected the vision and occlusion of retinal blood vessel, accompanied by optic atrophy in some patients. It was interesting to note that both mild and severe cases showed ocular manifestations. Severe cases were docu- 
mented in the accession files of the hospitals, rendering around 40,000 mildly affected people with no record 21 .
Details of suspected and confirmed cases of RVFV in Saudi Arabia in the last two decades are presented in Table 2.

Table 2: Outbreak of Rift Valley Fever Virus (RVFV) in the Kingdom of Saudi Arabia in the last two decades.

\begin{tabular}{|c|c|c|c|c|c|}
\hline $\begin{array}{c}\text { No of } \\
\text { suspected } \\
\text { cases } \\
\end{array}$ & $\begin{array}{c}\text { No } \\
\text { of confirmed } \\
\text { cases } \\
\end{array}$ & $\begin{array}{c}\text { No of } \\
\text { fatalities }\end{array}$ & Year & City & Reference \\
\hline 294 & 50 & $\mathrm{NA}^{1}$ & 1999 & Makkah & 44 \\
\hline 516 & 206 & 87 & 2000 & Jizan, Asir region & 45 \\
\hline 884 & NA & 124 & $\begin{array}{c}2000- \\
01\end{array}$ & Jizan \& Asir & 46 \\
\hline 165 & 99 & 56 & 2000 & Jizan & 23 \\
\hline 886 & 834 & 190 & $\begin{array}{c}2000- \\
01\end{array}$ & $\begin{array}{c}\text { Jizan, Asir \& Qun } \\
\text { fuda }\end{array}$ & 24 \\
\hline
\end{tabular}

${ }^{1} \mathrm{NA}=$ Not Applicable

\section{Effective management and future considerations}

Aggressive, active management is required to control the outbreak of the RVF. Detection and diagnosis in the early stage of an outbreak can minimise the detrimental effects of the virus and prevent its progression to the advanced stage. The initial serological confirmation of RVFV in a cluster of patients will pave the way in targeted surveillance to uncover more cases in the area of investigation. The serological facilities in the laboratories of Saudi Arabia are equipped to detect increased titer levels of $\operatorname{IgG}$ and $\operatorname{IgM}$ in the sera of the infected patients ${ }^{26}$.

Furthermore, the nucleic acid sequence of the RVFV can be determined following the amplification by the Polymerase Chain Reaction (PCR) technique ${ }^{27}$. Patients with advanced symptoms, such as encephalitis, must be subjected to examining the dynamics of RVFV specific-IgM levels in their cerebrospinal fluid. A recent se- roepidemiological study was performed on 389 children and adolescents from the regions of Jazan, Aseer and Al-Qunfuda, aiming at detection the RVFV-specific IgG and IgM. The study concluded the absence of RVFV infections, an indication of the success of the effective management of this disease by the dual efforts of the Saudi Ministry of Agriculture and Ministry of Health.

Awareness campaigns related to the modes of transmission and how to intercept against it have to be implemented in Saudi Arabia, targeting the sustainability of successful control. Simple measures could help in this control including, the use of insecticide-impregnated clothing, mosquito nets, avoiding the outdoor sleep, elimination of the breeding habitats of mosquitoes, effective disinfection, and routine surveillance. The resources must be available to execute strategies for development of a vaccine to immunise humans that 
live in endemic areas, and travelers visiting such areas. In an interesting study, Pittman et al., have evaluated the long-term immunogenicity induced by an inactivated RVF vaccine, concluding its safety and efficacy in preventing the symptoms of $\mathrm{RVF}^{28}$. Furthermore, the health of humans and animals are related to each other in the form of a sustainable relationship. Accordingly, the animals should be included in the vaccination campaign, an approach that is within the strategy of the 'One Health', approved by the World Animal Health Organization (Paris, France) and the World Health Organization (Geneva, Switzerland).

\section{Dengue Hemorrhagic Fever (DHF)}

Dengue is the most common and mosquito-borne viral disease, with potential transmission to nearly $50 \%$ of the population around the globe, especially in tropical and sub-tropical areas where it is entirely endemic $^{29}$. Consequently, this has led to a further significant rise in the healthcare economic burden, affecting the socioeconomic status of many countries. The history of the dengue-like symptoms dates back to the Wu dynasty $(222-280$ AD) and Dong dynasty (317 $420 \mathrm{AD})^{30}$. The vectors and the virus have expanded its distribution to different geographical areas, with a present hyper-endemicity in many parts of the world. It is reported that nearly 125 countries are endemic for dengue $^{34,35}$. The global incidence rate of dengue ranges from 50-200 millions new cases ${ }^{31}$. National surveillance in affected countries of the World is prerequisite to strategies targeting the dengue prevention and control programs established by the World Health Organization (WHO) (2012-2020). The first isolation of the Dengue virus in Saudi Arabia was performed in 1994 during a DHF outbreak in Jeddah, wherein 289 cases were confirmed $^{32}$. The first outbreak was caused by DENV-2; later investigations revealed high number of DENV-1 \& DENV-2 recovery that peaked in summer and late rainy seasons. More cases of DHF reemerged in Jeddah during year 1997 caused by DENV-3, while the years 2004-2006 had infections by DENV-1, DENV-2 \& DENV-336, ${ }^{38,39}$. The most recent episode of 2009 was reported by the $\mathrm{MOH}$, confirming the infection of 3350 cases by DHFV, associated with a fatality rate of 4.6 per thousand ${ }^{33}$.

\section{The diversity of Virus}

DHF is a vector-borne disease caused by a single positive-stranded RNA virus, referred to in the literature as DENV. DENV belongs to the genus, flavivirus of the family Flaviviridae which comprises nearly 70 viruses ${ }^{34}$. The Flaviviruses are spherical with a lipid envelope and size range of $40 \pm 50 \mathrm{~nm}$. Interestingly, all the flaviviruses have a common group epitope on the protein of the envelope which leads to cross-reactions in serological tests, creating a hindrance in the serological differential diagnosis among the viruses of DHF. Four different serotypes have been reported which are antigenically and phylogenetically distinct from each other ${ }^{34}$. In the Middle East countries, including Saudi Arabia and Yemen, three serotypes have been isolated until now, namely the DENV 1, DENV-2, and DENV-3. The DENV 1 isolated from Saudi Arabia has a high degree of genetic similarity to the DENV 1 isolated from Asian countries, suggesting an Asian genotype transmission through the pilgrims of Asia during their visit to Saudi Arabia ${ }^{42,43}$. It is worth noting that acquiring immunity to a serotype doesn't protect against the other serotypes. This requires development of vaccines specific to prevalent DHF serotypes of each country.

\section{Modes of transmission}

DHF is a vector-borne disease, transmitted by mosquito bites primarily those of Aedes aegypti and Aedes albopictus. The virus is transmitted from an infected patient to the healthy ones through the mosquitoes. Initially, the virus replicates in the epithelium of mid-gut of the mosquitoes, and after an incubation of 10-14 days, it spreads to different organs such as its salivary glands, allowing its transmission to blood of humans through the saliva of the mosquito bite. Many studies have reported the vertical transmission of the virus from infected mosquito to its larvae, and consequently to hatched mosquito offspring ${ }^{37}$. In a study performed in India, it was reported that the virus was infecting the larva, collected from different parts of rural and urban settings, using the indirect fluorescence antibody technique ${ }^{38}$. However, in humans the vertical transmission through blood or placenta from a DHFV-infected mother to its embryo is still controversial.

\section{Clinical challenge}

The infection with dengue virus elicits a spectrum of illnesses ranging from mild febrile illness to severe hemorrhagic manifestations. The incubation period of the virus varies from 3 to 14 days, depending on many factors. Moreover, the clinical symptoms in people inhabiting the endemic areas depend upon diverse factors including, the previous record of infection, immunity, age, serotype of the infecting virus, and the genetic 
background of the human and the vector. DHF occurs most commonly in children below the age of 15 , with reported occurrence in adults too. The incubation period is followed by sudden onset of fever that lasts for 2 to 7 days $^{34}$. Based on the data obtained from several recent studies, WHO has classified dengue into two cat-

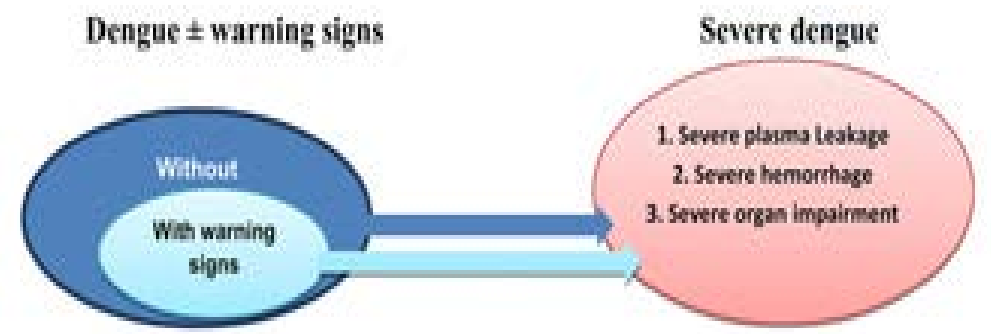

\section{Criteria for dengue \pm warning signs}

\begin{tabular}{|c|}
\hline 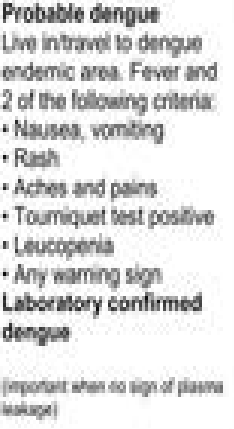 \\
\hline
\end{tabular}

\begin{tabular}{|c|}
\hline 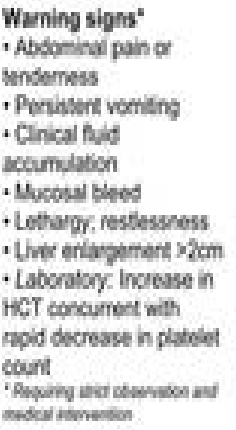 \\
\hline
\end{tabular}

\section{Criteria for severe dengue}

\begin{tabular}{|c|}
\hline 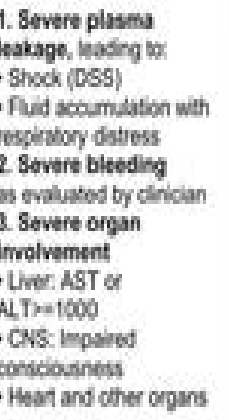 \\
\hline
\end{tabular}

Figure 3: Presumptive diagnosis of different Dengue stages based on specific symptoms. Adopted from 'Dengue Guidelines for diagnosis, treatment, prevention and control, New edn.,WHO/span>, Geneva, Switzerland, 2009'.

Additionally, patients showed decreased fibrinogen levels, thrombocytopenia, and a weak pulse. However, the patients with mild symptoms experienced profuse sweating, the coolness of the extremities and changes in blood pressure, which can be easily controlled by maintaining electrolyte balance. In a published study, the clinical profile of 160 suspected dengue patients, during an outbreak that occurred in Makkah between April to July of 2004, indicated that 75 patients had dengue fever, while only six patients suffered from DHF31. The details of suspected and confirmed cases of DHF in Saudi Arabia for the last two decades are presented in Table 3.

Table 3: Outbreak of Dengue fever virus in the Kingdom of Saudi Arabia in the last two decades.

\begin{tabular}{cccccc}
\hline $\begin{array}{c}\text { No of suspected } \\
\text { cases }\end{array}$ & $\begin{array}{c}\text { No of confirmed } \\
\text { cases }\end{array}$ & $\begin{array}{c}\text { No of } \\
\text { fatalities }\end{array}$ & Year & City & Reference \\
\hline 985 & 207 & 2 & $1994-99$ & Jeddah & 47 \\
197 & 1 & NA & 2000 & Jizan & 43 \\
80 & 39 & NA & $2004-05$ & Jeddah & 48 \\
160 & 91 & NA & 2004 & Makkah & 31 \\
690 & 151 & NA & $2010-15$ & Jeddah & 49 \\
\hline
\end{tabular}




\section{Effective management and future considerations}

Awareness campaign plays an essential role in a combined effort by all concerned organisations to reach to the effective management of the DHF contagious disease. A study published in 2008, assesed the knowledge related to dengue among teachers of high school and their students, concluding that nearly two-thirds of the surveyed were unaware that dengue is an infectious disease. Spraying of insecticides around the house, elimination of stagnant water, wearing clothing with long sleeves and using insecticide-impregnated bedding are some of the measures. Saudi Arabia took the appropriate steps, in collaboration with disease control centres and Ministry of health, imposing the guidelines of WHO concerning the One Health approach; however, these measures could fail due to the introduction of the Dengue virus by infected pilgrims that dwell in different Dengue-endemic countries ${ }^{39}$. Early detection and routine surveillances are needed to identify the rate of infectivity in populations of the various cities in the KSA and to help in evaluation of the effective management and control of this disease. A recent seroprevalence study, performed in Jeddah, indicated a presence of high exposure of the residents to dengue viruses ${ }^{40}$. Most of the infection were either mild or asymptomatic, requiring no treatment to these individuals; however, continued alertness and awareness are essential in reducing the progression to severe stages of the Dengue.

\section{Conclusion}

The infectious viral diseases should be given a priority in the healthcare sector, due to their impact on productivity and economy of the KSA, and the potential of their spread to other countries of the world. Moreover, the One Health approach, which aims at the improvement of the livelihood of humans and animals, and sustainability of the environment must be adopted in the strategy of control of these contagious viral diseases in the KSA. The regular immunisation of animals and humans is one component of the future strategy while maintaining strict quarantine on the imported livestock from endemic countries is another paramount component. Since mosquitoes are the vectors of many viruses; accordingly, the Saudi Ministry of Health and Ministry of Municipal Affairs have a responsibility to initiate an intensive insecticide control program, after concluding a thorough cleanliness procedure in the housing vicinity of the endemic areas. Finally, the regular interactions and exchange of knowledge, followed by preparation of new strategies in cooperation among health care workers, clinicians, virologists, epidemiologists, veter- inarians, WHO, and World Organisation For Animal Health will play an essential role in achieving the vision of creating a healthy nation which will also foster the economic growth of the country.

\section{Declaration of conflicting interests}

The author(s) have no relevant affiliations or financial involvement with any organisation or entity with a financial interest in or financial conflict with the subject matter or materials discussed in the manuscript. This includes employment, consultancies, honoraria, stock ownership, grants or patents received or pending and royalties. No writing assistance was utilised in the production of this manuscript.

No conflict of interest declared.

\section{Disclosures and ethics}

The data and material presented in this manuscript are neither published before nor have been submitted for publication to another scientific journal or is being considered for publication elsewhere. All the co-authors have read this manuscript and approved it for submission.

\section{References}

1. L. Ftika and H. C. Maltezou, "Viral haemorrhagic fevers in healthcare settings.," J. Hosp. Infect., vol. 83, no. 3, pp. 185-192, Mar. 2013.

2. A. M. Zaki, "Isolation of a flavivirus related to the tick-borne encephalitis complex from human cases in Saudi Arabia," Trans. R. Soc. Trop. Med. Hyg., vol. 91, no. 2, pp. 179-181, 1997.

3. A. G. Alzahrani et al., "Alkhurma Hemorrhagic Fever in Humans, Najran, Saudi Arabia," Emerg. Infect. Dis., vol. 16, no. 12, pp. 1882-1888, Dec. 2010.

4. Z. A. Memish, S. F. Fagbo, A. O. Ali, R. AlHakeem, F. M. Elnagi, and E. A. Bamgboye, "Is the epidemiology of alkhurma hemorrhagic fever changing?: A three-year overview in Saudi Arabia," PLoS One, vol. 9, no. 2, pp. 1-6, 2014.

5. A. Ul-Rahman, "Genetic diversity of Alkhurma hemorrhagic fever virus in Western Asia.," Infect. Genet. Evol., vol. 70, pp. 80-83, Jun. 2019.

6. Z. A. Memish et al., "Alkhurma viral hemorrhagic fever virus: proposed guidelines for detection, prevention, and control in Saudi Arabia.," PLoS Negl. Trop. Dis., vol. 6, no. 7, p. e1604, 2012.

7. R. N. Charrel et al., "Low Diversity of Alkhurma Hemorrhagic Fever Virus, Saudi Arabia, 1994-1999," Emerg. Infect. Dis., vol. 11, no. 5, pp. 683-688, May 2005. 
8. R. Mehla et al., "Recent ancestry of Kyasanur Forest disease virus," Emerg. Infect. Dis., vol. 15, no. 9, pp. 1431-1437, Sep. 2009.

9. R. N. Charrel et al., "Complete coding sequence of the Alkhurma virus, a tick-borne flavivirus causing severe hemorrhagic fever in humans in Saudi Arabia.," Biochem. Biophys. Res. Commun., vol. 287, no. 2, pp. 455461, Sep. 2001.

10. R. N. Charrel, S. Fagbo, G. Moureau, M. H. Alqahtani, S. Temmam, and X. de Lamballerie, "Alkhurma hemorrhagic fever virus in Ornithodoros savignyi ticks," Emerg. Infect. Dis., vol. 13, no. 1, pp. 153-155, Jan. 2007.

11. G. Grard et al., "Genetic characterization of tickborne flaviviruses: new insights into evolution, pathogenetic determinants and taxonomy.," Virology, vol. 361, no. 1, pp. 80-92, Apr. 2007.

12. M. Mahdi et al., "Kyasanur Forest Disease virus Alkhurma subtype in ticks, Najran Province, Saudi Arabia.," Emerging infectious diseases, vol. 17, no. 5. United States, pp. 945-947, May-2011.

13. Z. A. Memish, H. H. Balkhy, C. Francis, G. Cunningham, A. H. Hajeer, and M. A. Almuneef, "Alkhumra haemorrhagic fever: case report and infection control details.," Br. J. Biomed. Sci., vol. 62, no. 1, pp. 37-39, 2005.

14. T. A. Madani et al., "Successful propagation of Alkhumra (misnamed as Alkhurma) virus in C6/36 mosquito cells.," Trans. R. Soc. Trop. Med. Hyg., vol. 106, no. 3, pp. 180-185, Mar. 2012.

15. T. A. Madani et al., "Alkhumra (Alkhurma) virus outbreak in Najran, Saudi Arabia: epidemiological, clinical, and laboratory characteristics.," J. Infect., vol. 62, no. 1, pp. 67-76, Jan. 2011.

16. T. A. Madani, "Alkhumra virus infection, a new viral hemorrhagic fever in Saudi Arabia," J. Infect., vol. 51, no. 2, pp. 91-97, 2005.

17. J. A. Al-Tawfiq and Z. A. Memish, "Alkhurma hemorrhagic fever virus," Microbes Infect., vol. 19, no. 6, pp. 305-310, 2017.

18. S. Javed, F. Khan, M. Ramirez-Fort, and S. K. Tyring, "Bites and mites: prevention and protection of vector-borne disease.," Curr. Opin. Pediatr., vol. 25, no. 4, pp. 488-491, Aug. 2013.

19. T. P. Monath, "Vaccines against diseases transmitted from animals to humans: A one health paradigm," Vaccine, vol. 31, no. 46, pp. 5321-5338, 2013.

20. B. R. Miller et al., "Isolation and genetic characterization of Rift Valley fever virus from Aedes vexans arabiensis, Kingdom of Saudi Arabia," Emerg Infect Dis, vol. 8, no. 12, pp. 1492-1494, 2002.
21. A. I. Al-Afaleq and M. F. Hussein, "The Status of Rift Valley Fever in Animals in Saudi Arabia: A Mini Review," Vector-Borne Zoonotic Dis., vol. 11, no. 12, pp. 1513-1520, 2011.

22. P. G. Jupp et al., "The 2000 epidemic of Rift Valley fever in Saudi Arabia: mosquito vector studies.," Med. Vet. Entomol., vol. 16, no. 3, pp. 245-252, Sep. 2002.

23. M. Al-Hazmi et al., "Epidemic Rift Valley fever in Saudi Arabia: a clinical study of severe illness in humans.," Clin. Infect. Dis., vol. 36, no. 3, pp. 245-252, Feb. 2003.

24. T. A. Madani et al., "Rift Valley fever epidemic in Saudi Arabia: epidemiological, clinical, and laboratory characteristics.," Clin. Infect. Dis., vol. 37, no. 8, pp. 1084-1092, Oct. 2003.

25. A. Al-Hazmi et al., "Ocular complications of Rift Valley fever outbreak in Saudi Arabia.," Ophthalmology, vol. 112, no. 2, pp. 313-318, Feb. 2005.

26. O. D. Olaleye, O. Tomori, M. A. Ladipo, and H. Schmitz, "Rift Valley fever in Nigeria: infections in humans.," Rev. Sci. Tech., vol. 15, no. 3, pp. 923-935, Sep. 1996.

27. S. Garcia et al., "Quantitative real-time PCR detection of Rift Valley fever virus and its application to evaluation of antiviral compounds.," J. Clin. Microbiol., vol. 39, no. 12, pp. 4456-4461, Dec. 2001.

28. P. R. Pittman et al., "Immunogenicity of an inactivated Rift Valley fever vaccine in humans: a 12-year experience.," Vaccine, vol. 18, no. 1-2, pp. 181-189, Aug. 1999.

29. J. A. Al-Tawfiq and Z. A. Memish, "Dengue Hemorrhagic Fever Virus in Saudi Arabia: A Review," Vector-Borne Zoonotic Dis., vol. 18, no. 2, pp. 75-81, Jan. 2018. 30. D. J. Gubler, "Dengue/dengue haemorrhagic fever: history and current status.," Novartis Found. Symp., vol. 277, pp. 3-22,71-73,251-253, 2006.

31. N. A. Khan et al., "Clinical profile and outcome of hospitalized patients during first outbreak of dengue in Makkah, Saudi Arabia.," Acta Trop., vol. 105, no. 1, pp. 39-44, Jan. 2008.

32. M. Fakeeh and A. M. Zaki, "C," Am. J. Trop. Med. Hyg., vol. 65, no. 6, 2001.

33. M. of Health, "Health Statistical Year Book," 2009. 34. D. J. Gubler, "Dengue and Dengue Hemorrhagic Fever," Clin. Microbiol. Rev., vol. 11, no. 3, pp. 480-496, 1998.

35. T. A. Madani et al., "Outbreak of viral hemorrhagic fever caused by dengue virus type 3 in Al-Mukalla, Yemen," BMC Infect. Dis., vol. 13, no. 1, p. 136, 2013.

36. E. I. Azhar et al., "Complete genome sequencing and phylogenetic analysis of dengue type 1 virus iso- 
lated from Jeddah, Saudi Arabia," Virol. J., vol. 12, p. 1, Jan. 2015.

37. T. Nedjadi, S. El-Kafrawy, S. S. Sohrab, P. Desprès, G. Damanhouri, and E. Azhar, "Tackling dengue fever: Current status and challenges," Virol. J., vol. 12, p. 212, Dec. 2015.

38. B. Angel and V. Joshi, "Distribution and seasonality of vertically transmitted dengue viruses in Aedes mosquitoes in arid and semi-arid areas of Rajasthan, India.," J. Vector Borne Dis., vol. 45, no. 1, pp. 56-59, Mar. 2008. 39. O. A. Hassan, C. Ahlm, and M. Evander, "A need for One Health approach - lessons learned from outbreaks of Rift Valley fever in Saudi Arabia and Sudan.," Infect. Ecol. Epidemiol., vol. 4, pp. 1-8, 2014.

40. G. A. Jamjoom, E. I. Azhar, M. A. Kao, and R. M. Radadi, "Seroepidemiology of Asymptomatic Dengue Virus Infection in Jeddah, Saudi Arabia," Virology (Auckl)., vol. 7, pp. 1-7, Feb. 2016.

41. A. M. Zaki, "Isolation of a flavivirus related to the tick-borne encephalitis complex from human cases in Saudi Arabia.," Trans. R. Soc. Trop. Med. Hyg., vol. 91, no. 2, pp. 179-181, 1997.

42. T. A. Madani, "Alkhumra virus infection, a new viral hemorrhagic fever in Saudi Arabia.," J. Infect., vol. 51, no. 2, pp. 91-97, Aug. 2005.

43. Z. A. Memish et al., "Seroprevalence of Alkhurma and Other Hemorrhagic Fever Viruses, Saudi Arabia," Emerg. Infect. Dis., vol. 17, no. 12, pp. 2316-2318, Dec. 2011.
44. A. H. Turkistany, A. G. Mohamed, and N. Al-Hamdan, "Seroprevalence of Rift valley fever among slaughterhouse personnel in Makkah during Hajj 1419 H (1999).," J. Family Community Med., vol. 8, no. 3, pp. 53-57, Sep. 2001.

45. "Update: outbreak of Rift Valley Fever--Saudi Arabia, August-November 2000.," MMWR. Morb. Mortal. Wkily. Rep., vol. 49, no. 43, pp. 982-985, Nov. 2000.

46. T. Shoemaker et al., "Genetic analysis of viruses associated with emergence of Rift Valley fever in Saudi Arabia and Yemen, 2000-01.," Emerg. Infect. Dis., vol. 8, no. 12, pp. 1415-1420, Dec. 2002.

47. M. Fakeeh and A. M. Zaki, "Virologic and serologic surveillance for dengue fever in Jeddah, Saudi Arabia, 1994-1999.," Am. J. Trop. Med. Hyg., vol. 65, no. 6, pp. 764-767, Dec. 2001.

48. M. Ayyub, A. M. Khazindar, E. H. Lubbad, S. Barlas, A. Y. Alfi, and S. Al-Ukayli, "Characteristics of dengue fever in a large public hospital, Jeddah, Saudi Arabia.," J. Ayub Med. Coll. Abbottabad, vol. 18, no. 2, pp. 9-13, 2006.

49. M. S. Al-Saeed et al., "Phylogenetic characterization of circulating Dengue and Alkhumra Hemorrhagic Fever viruses in western Saudi Arabia and lack of evidence of Zika virus in the region: A retrospective study, 20102015.," J. Med. Virol., vol. 89, no. 8, pp. 1339-1346, Aug. 2017. 